\title{
Secure and Optimized Cloud-Based Cyber-Physical Systems with Memory-Aware Scheduling Scheme
}

\author{
Dr. Wang Haoxiang ${ }^{1}$, Dr. S. Smys ${ }^{2}$ \\ ${ }^{1}$ Director and lead executive faculty member, \\ GoPerception Laboratory, \\ NY, USA. \\ ${ }^{1}$ Email: hw496@goperception.com \\ ${ }^{2}$ Professor, Department of CSE, \\ RVS Technical Campus, \\ Coimbatore, India. \\ 2Email: smys375@gmail.com
}

\begin{abstract}
Computer based algorithms are used for monitoring and controlling a computers where human, physical, digital and analog interaction occurs. This scheme termed as cyber-physical systems (CPS) is being deployed in a wide array of applications due to its easy deployment feature and connectivity. CPS based on cloud computing technology offers wider application range with enormous amount of storage and computing resources. However, despite the wide application and deployment of CPS in combining the key technologies like big data analytics, cloud computing and IoT, its energy consumption is large. Optimization of this energy is a major field of research and is of due importance. For this purpose, in cloud environment, virtual machines (VMs) are used for hosting the applications and the resources are managed thereby optimizing the energy consumption. The security issues and the quality of service (QoS) requirements of the system is also met by optimizing the system design. The scheduling issue of the system is addressed by implementation of efficient memory-aware scheduling strategy and algorithms. The proposed technique is tested for performance and the results of simulation is presented.
\end{abstract}

Keywords: Security; Energy Efficiency; Cyber-physical systems; Cloud storage; Memory-aware scheduling; Quality of Service; Virtual Machines;

\section{Introduction}

Cyber-physical Systems have evolved rapidly in various critical domains like traffic control, manufacturing, healthcare and so on due to its efficient and timely control, management and data evaluation capabilities [1]. For the implementation of distributed computing resources, CPS technology is used by several enterprises globally. The real world data is collected by the sensors and transmitted for analysis by the computation platforms. A command or feedback is returned to the system after analysis of the data. Efficient decision making ability of the system is influenced by the real-time data provided by the CPS [2]. Information is obtained from multiple sources with the integration of mobile and CPS with the rapid advancement of technology. The user performance requirements are met by the massive computing resources and storage available in complex CPS applications. However, the limited computing and storage capacity of mobile devices are a challenge to the efficiency of CPS. This is overcome by using cloud computing and storage solutions that offer large computing and storage space for all applications [3]. 
Journal of trends in Computer Science and Smart technology (TCSST) (2020)

Vol.02/ No. 03

Pages: $141-147$

https://www.irojournals.com/tcsst/

DOI: https://doi.org/10.36548/jtcsst.2020.3.003

The resource driven applications make use of the rich cloud resources for improving the computational capacity of the CPS [4]. The cloud platform resource management is extensively performed by the virtualized technology and dynamic physical resources, thereby improving the cloud CPS resource efficiency in an effective manner. Low energy consumption and high resource utilization is made possible by implementation of virtual machines (VMs) for running applications [5]. Integration of cloud storage automobiles, mobile phones and several applications that generate large volume of data with limited storage and computational space with the CPS technology improves the efficiency and the resource limit to a greater extent [6].

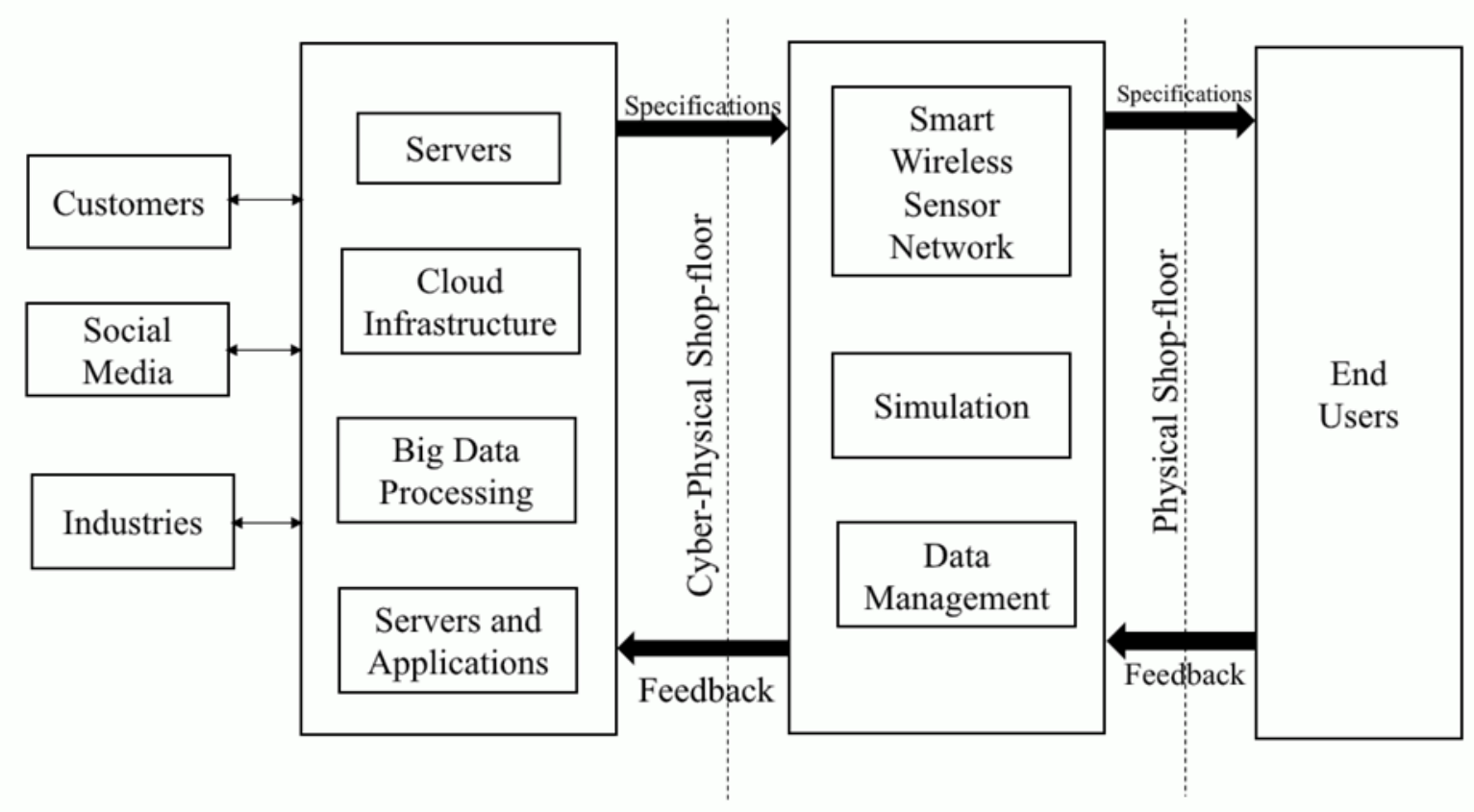

Figure 1: CPS Conceptual Framework Schematic Diagram

For improving the cloud-based CPS services, it is essential to efficiently migrate the applications to the cloud. For this purpose, several scheduling strategies are tested and implemented. The VM migration technique offers several advantages, however, the datacenter switches cost additional consumption of energy and transmission delay [7]. Hence, formulation of a reasonable scheduling strategy involves taking into account the positive and negative features as well as various user requirements for VM migration. The battery life of the system also reduces severely as the cloud providers operation cost is augmented by cloud-based CPS. The resource intensive applications are restricted for user experience in cloud-based data-centers thereby reducing the energy consumption of the system [8].

\section{Literature Review}

The intermediate layer between the cyber computing and physical factory layer systematically makes use of CPS in the manufacturing industries [9]. Quality, reliability and efficiency of the CPS architecture are the major parameters to be considered during the design of the system [10]. Intelligent management of data and advanced 
Journal of trends in Computer Science and Smart technology (TCSST) (2020)

Vol.02/ No. 03

Pages: $141-147$

https://www.irojournals.com/tcsst/

DOI: https://doi.org/10.36548/jtcsst.2020.3.003

connectivity are the major components of the proposed structure of CPS along with secure and fast processing. The complexity of the system is managed by segregation and aspect-oriented modeling by addressing the multidisciplinary nature, heterogeneity and complexity of the industrial CPS [11]. The implementation and contribution of CPS in smart grid development and the potential advancements are analyzed by several researchers. Advanced communication, control and monitoring efficiency is offered by the CPS system. For successful monitoring of industrial applications, several challenges such as lacking performance in dynamic and real-time conditions are imposed by the CPS. Wireless control and cyber-physical system co-design, wireless cyber physical simulation, real-time scheduling algorithms are some of the industrial applications of CPS in real-time wireless networks [12].

The mobile device limit capacity is overcome by using the cloud for offloading the resource intensive applications [13]. The network quality is a major factor that is to be considered while offloading the applications. Remote public cloud and nearby cloudlets make use of different varieties of offloading frameworks that is developed by various researchers based on the applications. Offloading decisions are provided for adaptive code in the mobile device context by using cloud for offloading. Long latency and interference is produced when offloading is done by multiple users simultaneously into the cloud environment. Reasonable and efficient decisions are made for offloading using game theory and dynamic computation offloading techniques for multiple users. The context of the perspective user is used for improving the quality of experience of the user [14]. A tradeoff between cost, latency and energy is presented for solving the scheduling issue and selection of services for offloading.

\section{Problem Formulation}

The memory aware VM scheduling technique and the formalized concepts of the system is introduced with respect to the cloud-based CPS. Throughput, resource utilization, availability, response time, energy consumption and such nonfunctional requirements are analyzed and quantified. The optimization, speed as well as security issue of the system is addressed as a multi-objective optimization issue. This paper works on providing an optimal solution to this issue by means of memory aware VM scheduling.

\section{Proposed Work}

The multi-objective optimization issue is overcome by adopting suitable techniques to overcome the speed, security and other significant errors of the system. Multiple objectives between 3-15 are analyzed while solving such issues. This helps in improving the performance accuracy and efficiency of the system. The fitness function and encoding strategies are analyzed in VM scheduling and a suitable algorithm is adopted for optimization of the solution. Memory aware cloud scheduling scheme enables fast and error free scheduling and improves the security of the system 
Journal of trends in Computer Science and Smart technology (TCSST) (2020)

Vol.02/ No. 03

Pages: $141-147$

https://www.irojournals.com/tcsst/

DOI: https://doi.org/10.36548/jtcsst.2020.3.003

\subsection{Memory-Aware Cloud Scheduling}

Each VM collects data from the physical machine regarding cache behavior by means of the scheduler in memory aware scheduling scheme. This improves the accuracy of the output by significantly reducing the memory access latencies and the overall cache misses in the cloud environment. The monitoring counters of hardware performance offers a monitor check for the cache misses in each PM. The data is further sent to the cloud scheduler on a periodic basis. Global scheduling decisions are made by the cloud scheduler based on the status information obtained by the VM from all nodes. There is a considerable rise in the number of migrations despite the memory contention reduction between the same host VMs by the memory aware schedulers. The global and local phases are used by cache-aware scheduler for scheduling the behavior of cache in all nodes or specific nodes.

\section{Results and Discussion}

\subsection{Simulation setup}

Four diverse data sets with unique scales for various applications are used for simulation purpose with different number of applications. Table 1 provides the initial parameter settings used in the experiment. The simulation results are monitored based on the energy consumption, efficiency and downtime.

Table 1: Parameter Settings

\begin{tabular}{|l|l|}
\hline \multicolumn{1}{|c|}{ Parameter } & \multicolumn{1}{c|}{ Value } \\
\hline Total Applications Testes & 50 \\
\hline Physical Machines involved & 500 \\
\hline Rate of energy consumption in physical machines & $430 \mathrm{~W}$ \\
\hline Rate of energy consumption in virtual machines & $25 \mathrm{~W}$ \\
\hline
\end{tabular}

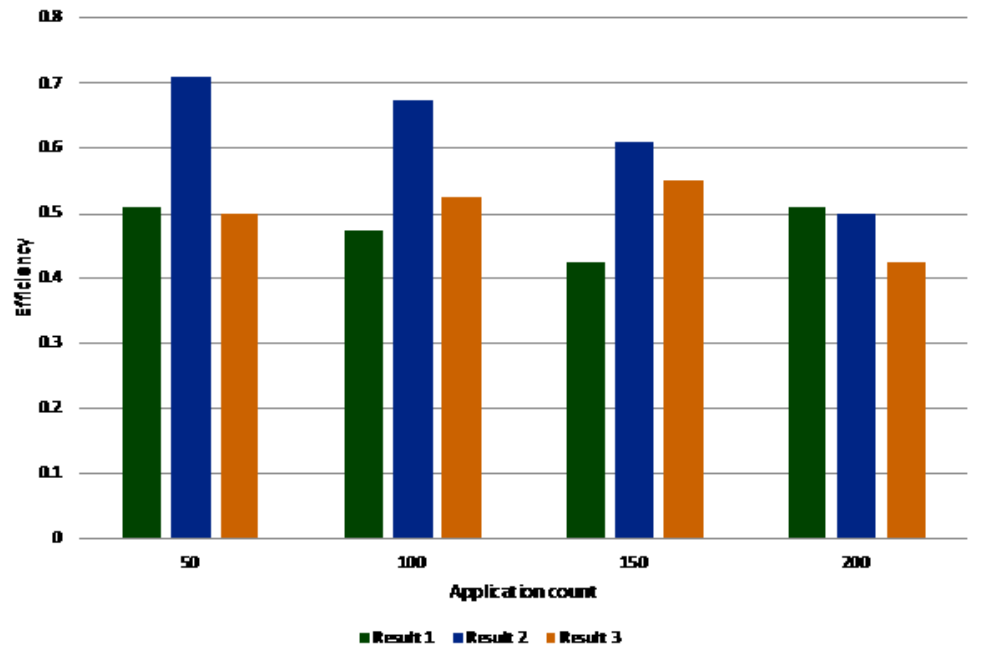

Figure 2: Comparison of Efficiency 
Journal of trends in Computer Science and Smart technology (TCSST) (2020)

Vol.02/ No. 03

Pages: $141-147$

https://www.irojournals.com/tcsst/

DOI: https://doi.org/10.36548/jtcsst.2020.3.003

\subsection{Performance analysis of Memory Aware Virtual Machine Scheduling Scheme}

Different numbers of applications are tested for generation of solutions based on QoS aware, energy aware and memory aware virtual machine systems as represented in figure 3. This analysis of the generated solutions provides the most optimal scheduling strategy on comparison of a standard utility value. Higher the utility value, more optimal the scheduling strategy is. On comparison, it is evident that the memory aware virtual machine scheduling offers the most optimal solution.

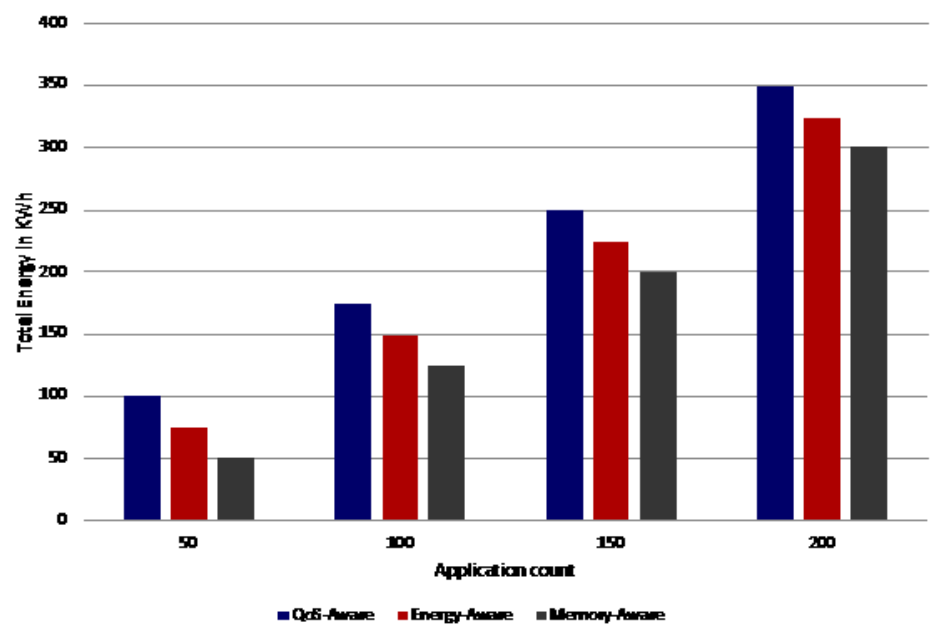

Figure 3: Comparison of Total Energy Utilization

The packet forwarding scheme performance for virtual machines is evaluated by computing the deadline miss ratio. This enables secure and fast scheduling. The sensor nodes are deployed randomly and varied for testing the deadline miss ratio. The random sensor node deployment and varying sensor nodes with respect to the deadline miss ratio is represented in Figure 4. A 20 millisecond deadline and 10 minute simulation time is used for experimentation. Packet transmission is performed by each source at a 1 second interval. As the node count of the network increases, the deadlines are not met by the packets as represented by the graph. When the sensor nodes are deployed in the grid, this behavior can be observed clearly. The non-uniform pattern in random deployment offers flexibility of usage of this behavior. 
Journal of trends in Computer Science and Smart technology (TCSST) (2020)

Vol.02/ No. 03

Pages: $141-147$

https://www.irojournals.com/tcsst/

DOI: https://doi.org/10.36548/jtcsst.2020.3.003

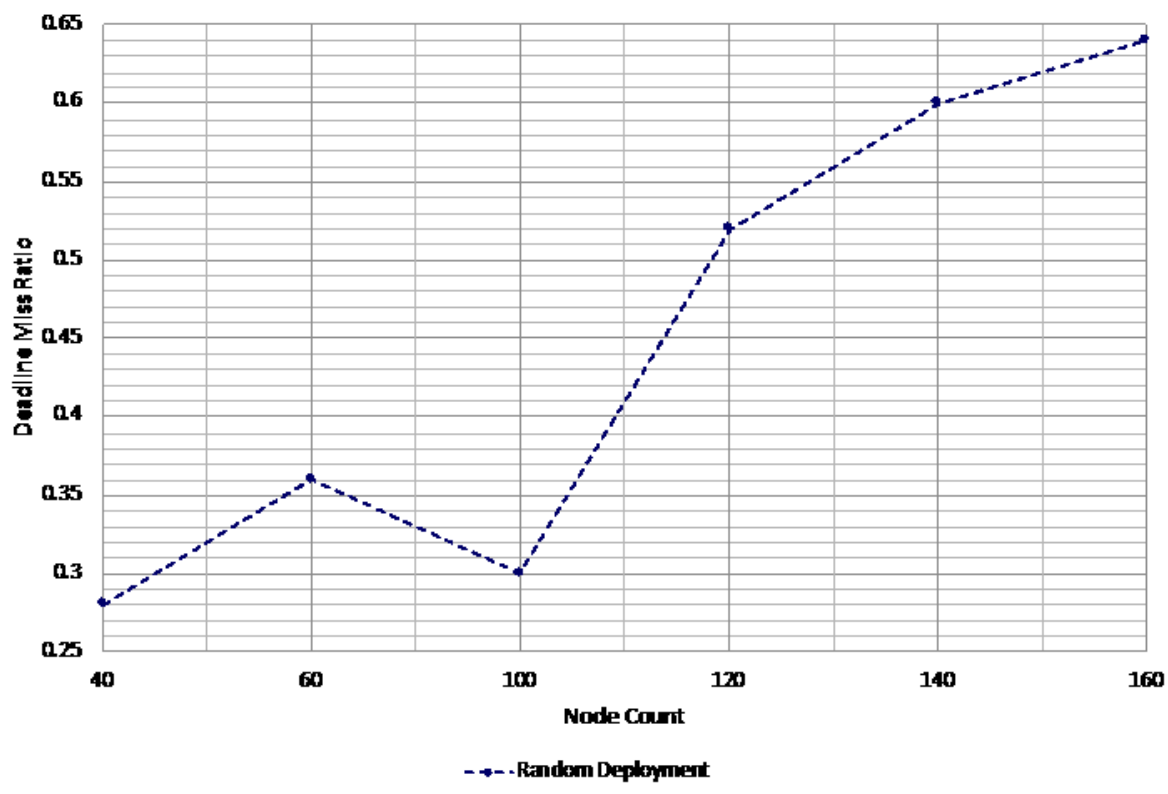

Figure 4: Deadline Miss Ratio Analysis

\section{Conclusion}

Cloud-based CPS is implemented with memory-aware virtual machine scheduling for secure and efficient operation in this paper. Energy consumption, deadline miss ratio and resource utilization are the parameters analysed using this systematic model. The problems related to security is also addressed by this system thereby providing a multi-objective optimization. The memory aware cloud scheduling scheme and optimization algorithm are analysed and compared with other scheduling schemes. This comparison proves the efficiency of the proposed technique. The system is also designed in such a way that it offers flexibility to the requirements of multiple users. Future work is focused on real-time implementation and analysis of the proposed model.

\section{References}

[1] Roy, D., Zhang, L., Chang, W., Mitter, S. K., \& Chakraborty, S. (2017). Semantics-preserving cosynthesis of cyber-physical systems. Proceedings of the IEEE, 106(1), 171-200.

[2] Toussaint, W., \& Ding, A. Y. (2020). Machine Learning Systems for Smart Services in the Internet of Things. arXiv preprint arXiv:2006.04950.

[3] Kurdi, H. A., Alismail, S. M., \& Hassan, M. M. (2018). LACE: A locust-inspired scheduling algorithm to reduce energy consumption in cloud datacenters. IEEE Access, 6, 35435-35448.

[4] AlIsmail, S. M., \& Kurdi, H. A. (2016). Review of energy reduction techniques for green cloud computing. Int. J. Adv. Comput. Sci. Appl, 1, 189-195.

[5] Vaquero, L. M., Cuadrado, F., Elkhatib, Y., Bernal-Bernabe, J., Srirama, S. N., \& Zhani, M. F. (2019). Research challenges in nextgen service orchestration. Future Generation Computer Systems, 90, 20-38. 
Journal of trends in Computer Science and Smart technology (TCSST) (2020)

Vol.02/ No. 03

Pages: $141-147$

https://www.irojournals.com/tcsst/

DOI: https://doi.org/10.36548/jtcsst.2020.3.003

[6] Kaur, T., \& Chana, I. (2015). Energy efficiency techniques in cloud computing: A survey and taxonomy. ACM computing surveys (CSUR), 48(2), 1-46.

[7] Alam, K. M., \& El Saddik, A. (2017). C2PS: A digital twin architecture reference model for the cloudbased cyber-physical systems. IEEE access, 5, 2050-2062.

[8] Majstorovic, V. D., Durakbasa, N. M., Mourtzis, D., \& Vlachou, E. (2016). Cloud-based cyber-physical systems and quality of services. The TQM Journal.

[9] Sanislav, T., Mois, G., Folea, S., Miclea, L., Gambardella, G., \& Prinetto, P. (2014, June). A cloud-based Cyber-Physical System for environmental monitoring. In 2014 3rd Mediterranean Conference on Embedded Computing (MECO) (pp. 6-9). IEEE.

[10] Reddy, Y. B. (2014, December). Cloud-based cyber physical systems: Design challenges and security needs. In 2014 10th International Conference on Mobile Ad-hoc and Sensor Networks (pp. 315-322). IEEE.

[11] Shakya, S. (2020). Survey on Cloud Based Robotics Architecture, Challenges and Applications. Journal of Ubiquitous Computing and Communication Technologies (UCCT), 2(01), 10-18.

[12] Karunakaran, V. (2019). A STOCHASTIC DEVELOPMENT OF CLOUD COMPUTING BASED TASK SCHEDULING ALGORITHM. Journal of Soft Computing Paradigm (JSCP), 1(01), 41-48.

[13] Mugunthan, S. R. (2019). SOFT COMPUTING BASED AUTONOMOUS LOW RATE DDOS ATTACK DETECTION AND SECURITY FOR CLOUD COMPUTING. Journal of Soft Computing Paradigm (JSCP), 1(02), 80-90.

[14] Mourtzis, D., \& Vlachou, E. (2018). A cloud-based cyber-physical system for adaptive shop-floor scheduling and condition-based maintenance. Journal of manufacturing systems, 47, 179-198. 